\title{
DADOS ECOLÓGICOS DOS QUIRÓPTEROS DA RESERVA VOLTA VELHA, ITAPOÁ, SANTA CATARINA, BRASIL
}

\author{
Elenise A. Bastos Sipinski ${ }^{1}$ \\ Nélio Roberto dos Reis ${ }^{2}$
}

\begin{abstract}
ECOLOGICAl. DATA OF ChIROPTERA From RESERVA VOLTA VElHA, ItapoA. Santa Cantakina. BRaZil. A study on the ecology of Chiroptera was made at the Reserve of Volta Velha on the Northern coast of Santa Catarina. This study aimed at knowing the frequency of the individuals collected in each season. part of their feeding diet and their time of activity as well as their breeding activity. The investigation was carried out from March, 1990 to February. 1991 by means of periodical captures which ocurred during four days each month totalizing 144 hours of net use. One hundred and thirty-five individuals belonging to 15 different species were caught. Stumira lilium (Geoffroy. 1810). Artibeus lituratus (Olfers, 1818) and Myotis nigricans (Schinz, 1821) were captured in the highest number throughout the seasons of the year. Fruit-eating bats confirm their feeding preference for Cecropiaceac, Solanaceac. Myrtaceae and Piperaceae. The hirths occurred mostly in seasons other than the dry. Around 1:15 hours after sunset was the time when the highest number of individuals were collected in the nets.
\end{abstract}

KEY WORDS. Chiroptera, ecology, bats, Atlantic forest, Brazil

O intenso desenvolvimento agrícola e agropecuário em Santa Catarina (Brasil), sobretudo nos últimos 60 anos, modificou o aspecto da vegetação primária dessa região (KLEIN 1978). Apesar da exploração desordenada, existem ainda núcleos florestais que permanecem intactos. A Reserva Volta Velha, que faz parte da Fazenda Palmital (Itapoá, Garuva), é um desses núcleos e está situada no litoral norte de Santa Catarina. É uma propriedade particular destinada à conservação, tendo sido enquadrada como Reserva Particular do Patrimônio Natural em 1990, abrigando uma grande diversidade da fauna e da flora local.

Dentre as espécies de animais existentes nesta região, encontram-se os quirópteros, que desempenham papel muito importante no ambiente, interferindo diretamente no controle de insetos (PINE 1968; HOWEL \& BURCH 1974; Kunz \& WHITAKER 1974; FABIAN et al. 1990), já que um morcego chega a comer centenas de insetos em menos de 60 minutos (GRIFFIN et al. 1960). Os quirópteros estão também relacionados com outros fenômenos intimamente ligados à composição florística das florestas tropicais, tais como a polinização, observada por Carvalho (1960), Sazima \& Sazima (1975, 1978) Sazima et al. (1982). Foi

1) Sociedade de Pesquisa na Vida Selvagem. Caixa Postal 305, 80420-030 Curitiba, Paraná, Brasil.

2) Departamento de Biologia Animale Vegetal. Universidade Estadual de Londrina. Caixa Postal 6001. 86051-970 Londrina. Paraná, Brasil. 
estimado também, que os quirópteros polinizam cerca de 500 espécies em 96 diferentes gêneros de plantas neotropicais (VOGEL 1969). De acordo com GreENhall (1966), Smythe (1970), Pul (1972), Uieda \& Vasconecellos-Neto (1985), esses animais são dispersores de sementes, chegando até ser sugerido por Humphrey \& BONACCORSO (1979), que um quarto das árvores de algumas florestas são dispersadas por eles.

Trabalhos que abordam a ecologia de quirópteros em Santa Catarina, praticamente inexistem. O presente trabalho visa fazer um levantamento das espécies existentes, conhecer a freqüência dos indivíduos coletados em cada estação, conhecer parte de sua dieta, bem como obter dados relativos à reprodução e horário de atividade dos indivíduos coletados.

\section{MATERIAL E MÉTODOS}

O estudo foi desenvolvido entre março de 1990 e fevereiro de 1991, quando foram realizadas coletas periódicas durante quatro dias de cada mês, totalizando 144 horas.

A técnica de captura foi baseada em Greenhall \& Paradiso (1968) e REIS (1981), com utilização de aproximadamente quatro a cinco redes-neblina (mist-nets), com dimensões variando de 5 a 18 metros de comprimento por dois metros de altura, totalizando $1.563 \mathrm{~m} /$ ano de redes. Estas foram armadas em diferentes pontos da Reserva: em locais abertos (pastagem), clareiras, trilhas, estradas e transversalmente em cursos de rio.

Para colher dados sobre a alimentação das espécies, foram obtidas amostras de fezes, ora na própria rede imediatamente após a captura do exemplar, ora em sacos de algodão, onde o espécime era deixado para defecar. O primeiro método mostrou ser mais eficiente para os frugívoros, provavelmente pelo fato da digestão destas espécies ser extremamente rápida (GLAss 1970), ou ainda pelo stress provocado pela captura.

Foi avaliado o estágio de vida de cada exemplar através da observação das articulações da asa, se ossificada no adulto e não totalmente ossificada no jovem. O estado reprodutivo foi observado nas fêmeas, através de apalpação no abdômen para constatar possível prenhez, e também através de sinais de lactação. Nos machos observou-se a presença ou não de testículo na bolsa escrotal.

Durante as coletas as redes eram verificadas, a cada 15 minutos, possibilitando registrar o horário de captura de cada indivíduo. O horário das coletas variou desde o entardecer até as três primeiras horas da noite, uma vez que os quirópteros neotropicais apresentam maior atividade externa, com pico nas primeiras horas escuras (BRown 1968; LAVAL 1970, FlEMING et al. 1972). As coletas foram feitas preferencialmente em noites sem luar, quando, segundo TAMSITT \& VALDIVIESO (1961) e MORRISON (1978), os morcegos ficam mais tempo fora de seus abrigos.

Três exemplares capturados de cada espécie foram fixados e conservados segundo orientação de VIZOTTO \& TADDEl (1973) e identificados segundo critérios de Vieira (1942), GoOdwin \& Greenhall (1961), Husson (1962), VizotTo \& TADDEI (1973) e JONES \& CARTER (1977). 


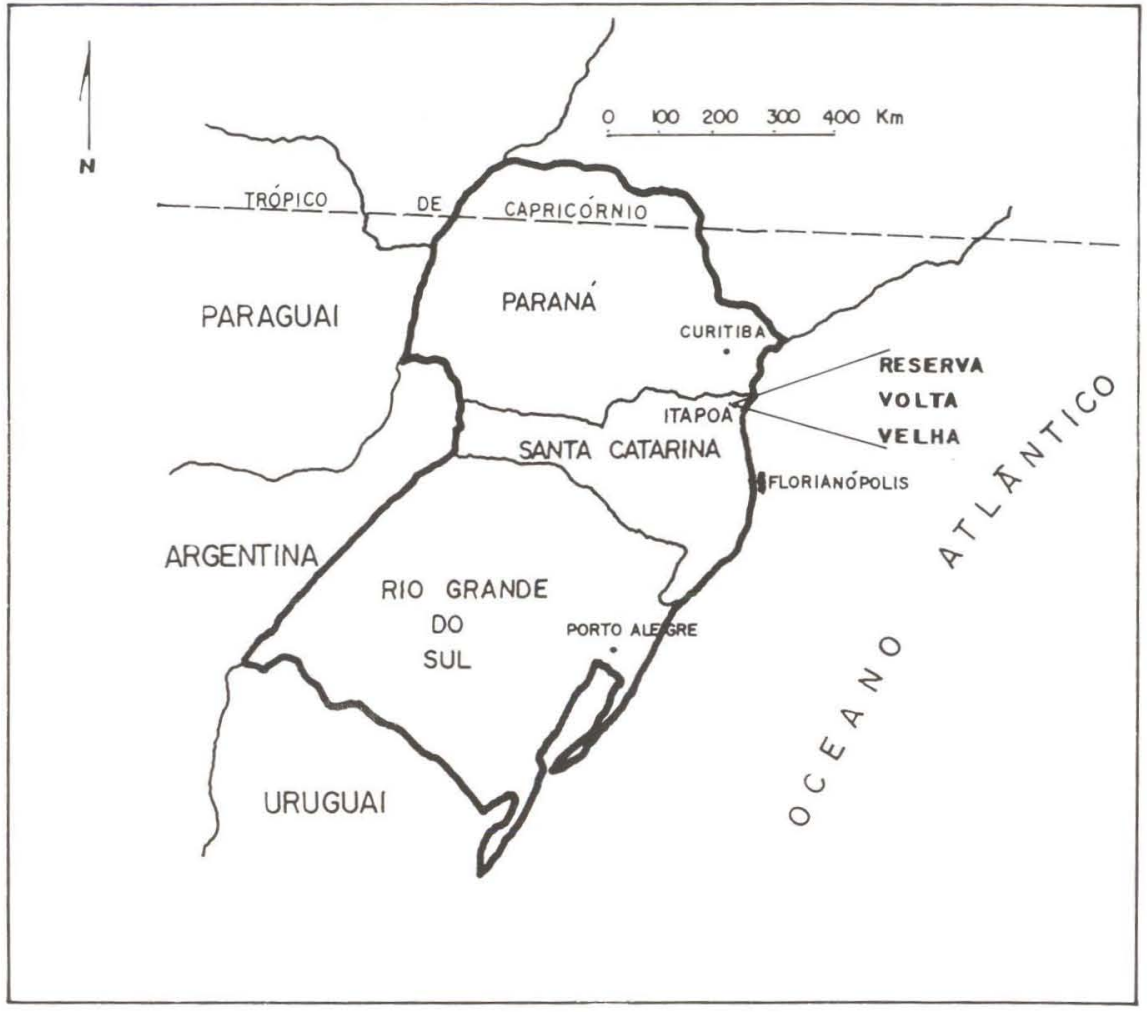

Fig. 1. Localização da Reserva Volta Redonda, Itapoá, Santa Catarina (1.186 hectares).

\section{DESCRIC̣ÃO DA ÁREA DE ESTUDO}

O estudo desenvolveu-se em uma área de floresta ombrofila densa de planície quaternária, localizada na Reserva Particular do Patrimônio Natural Volta Velha, na Fazenda Palmital (1.186 hectares). Tal área é situada no Município de Itapoá em Santa Catarina, (26 $04^{\circ} 05^{\prime \prime} \mathrm{S}, 48^{\circ} 37^{\prime} 30^{\prime \prime} \mathrm{W}$ ) (Fig. 1), quase divisa com o Paraná, aproximadamente a $5 \mathrm{Km}$ dos contra-fortes da Serra do Mar. Essa formação florestal na qual a propriedade está contida, representa uma larga faixa da planície costeira, que se estende desde o munícipio de Guaratuba (Paraná), até a boca do Rio Itapocú (Santa Catarina). Possui um tipo bem característico de mata, bastante uniforme quanto à sua composição, bem como quanto ao seu aspecto fisionômico.

Trata-se, em sua maior parte, de floresta primária, com pequenas variações altitudinais, distinguindo basicamente dois tipos de formações descritos por KLEIN (1978) como Tabuleiro (7-12 metros de altitude), caracterizado pela abundância de capiúva - Tapirira guianensis Aubl. (Anacardiaceae), Canela-sassafrás - Ocotea pretiosa (Ness) Mez (Lauraceae), canela amarela - Ocotea aciphylla (Ness et Mart. ex Nees (Lauraceae), canela-garuva - Nectandra rigida (H.B.K.) Ness (Lauraceae), guanandi - Calophyllum brasiliensis Camb. (Clusiaceae), tanheiro - 
Alchornea triplinervia (Sprengel) Mueller Arg. (Euphorbiaceae) e figueira - Ficus organensis (Miq.) Miq. (Moraceae), que dá um aspecto homogêneo e bastante característico à mata. A outra situa-se nas depressões do terreno (menos de sete metros de altitude), onde há pequenos cursos de águas, sendo caracterizado pela abundância de pau-de-santa-rita - Richeria australis Mueller Arg. (Euphorbiaceae), árvore exclusiva da zona norte das planícies do litoral. No estrato médio dessas matas predominam os seguintes arbustos: baga-de-morcego - Guarea macrophylla Vahl (Meliceae), seca-ligeiro - Pera glabrata (Schott) Bailon (Euphorbiaceae) e con-con - Erythroxylum amplifolium (Mart.) O.E. Schulz (Erythroxylaceae), caracterizando assim esse tipo de floresta.

\section{RESULTADOS}

Em 12 meses de coletas, foram capturados 134 indivíduos envolvendo 15 espécies. Artibeus lituratus (Olfers, 1818), foi a espécie mais coletada, seguida de Myotis nigricans (Schinz, 1821) e Sturnira lilium (Geoffroy, 1810). Esse quirópteros foram encontrados nas quatro estações do ano.

O maior número de espécies e de indivíduos foi coletado no inverno, seguido de verão e outono com números aproximados (Tab. I).

Tabela I. Espécies de morcegos coletados e sua freqüência nas diferentes estações do ano.

\begin{tabular}{|c|c|c|c|c|c|c|}
\hline Espécies & Outono & Inverno & Primavera & Veräo & Total & Porcentagem \\
\hline \multicolumn{7}{|l|}{ PHYLLOSTOMIDAE } \\
\hline Anoura caudifer (E.Geoffroy, 1818) & 1 & 3 & & & 4 & 2,9 \\
\hline Carollia perspicillata (L., 1758) & & 2 & & 1 & 3 & 2,2 \\
\hline Sturnira lilium (E. Geoffroy, 1810 ) & 2 & 5 & 7 & 2 & 16 & 11,8 \\
\hline Chiroderma doriae Thomas, 1891 & 13 & 2 & & & 15 & 11,1 \\
\hline Artibeus lituratus (Olfers, 1818) & 7 & 12 & 7 & 20 & 46 & 34,0 \\
\hline Artibeus fimbriatus Gray. 1838 & 6 & & & & 6 & 4,4 \\
\hline Artibeus jamaicensis Leach, 1821 & & 2 & 3 & 3 & 8 & 5,9 \\
\hline Pygoderma bilabiatum (Wagner, 1843) & 7 & 1 & & & 8 & 5,9 \\
\hline \multicolumn{7}{|l|}{ VESPERTILIONIDAE } \\
\hline Myotis nigricans (Schınz, 1821 ) & 2 & 7 & 4 & 7 & 20 & 14,8 \\
\hline Myotis ruber (E. Geoffroy, 1806) & & & 1 & & 1 & 0,7 \\
\hline Eptesicus brasiliensis (Desmarest, 1819) & & 1 & & & 1 & 0.7 \\
\hline Lasiurus borealis (Müller, 1776) & & 1 & & & 1 & 0.7 \\
\hline Lasiurus ega (Gervais, 1856) & & 1 & & 1 & 2 & 1,4 \\
\hline \multicolumn{7}{|l|}{ MOLOSSIDAE } \\
\hline Molossus molossus (Pallas, 1766) & 1 & & & 1 & 2 & 1,4 \\
\hline Molossus ater E. Geoffroy, 1805 & 1 & & & & 1 & 0,7 \\
\hline TOTAL & 33 & 43 & 23 & 35 & 134 & 100,0 \\
\hline
\end{tabular}

Quanto à alimentação, os dados mais expressivos são os relativos aos morcegos frugívoros (Tah. II). Carollia perspicillata (Linnaeus, 1758) com exclusividade sobre as Piperaceae, já $A$. lituratus e $S$. lilium mostram um aspecto maior na alimentação. Além dos animais citados na tabela II, foram encontradas uma espécie polinívora e sete insetívoras. 


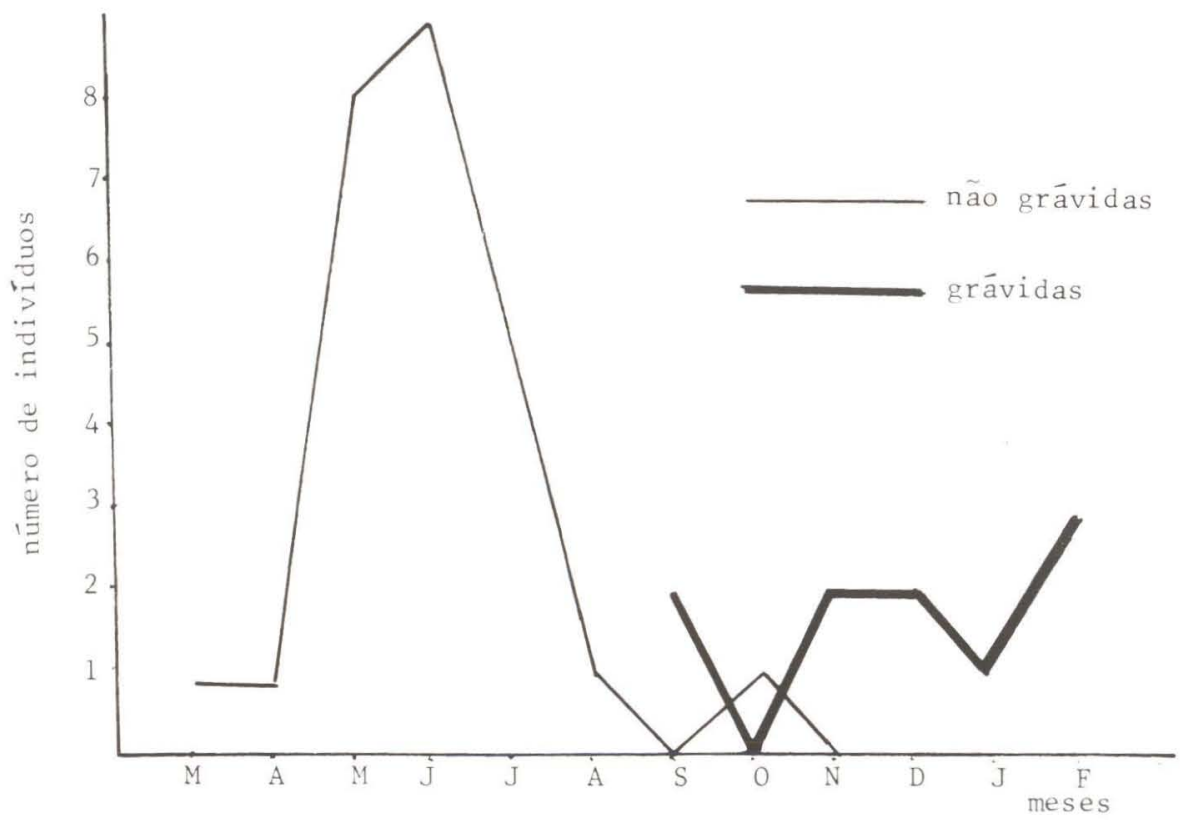

Fig. 2. Número de fêmeas grávidas e não grávidas no transcorrer das coletas.

Tabela II. Dados sobre a alimentação dos morcegos frugívoros.

\begin{tabular}{ll}
\hline \multicolumn{1}{c}{ Espécie de Phylostomidae } & \multicolumn{1}{c}{ Espécies vegetais } \\
\hline Carollia perspicillata & Piper sp. \\
Sturnira lilium & $\begin{array}{l}\text { Cecropia adenopus } \\
\text { Solanum sp. } \\
\text { Myrtaceae }\end{array}$ \\
Artibeus lituratus & $\begin{array}{l}\text { Cecropia adenopus } \\
\text { Ficus organensis }\end{array}$ \\
Artibeus jamaincensis & Cecropia adenopus \\
Chiroderma doriae & Myrtaceae \\
Pygoderma bilabiatum & Ficus organensis \\
\hline
\end{tabular}

Os dados sobre o estágio reprodutivo dos espécimes coletados encontram-se na tabela III. Em relação aos frugívoros, houve um pico maior de fêmeas grávidas de novembro a fevereiro e de fêmeas não grávidas de março a julho (Fig. 2).

O horário de maior atividade dos morcegos foi uma hora após o entardecer (Fig. 3). 


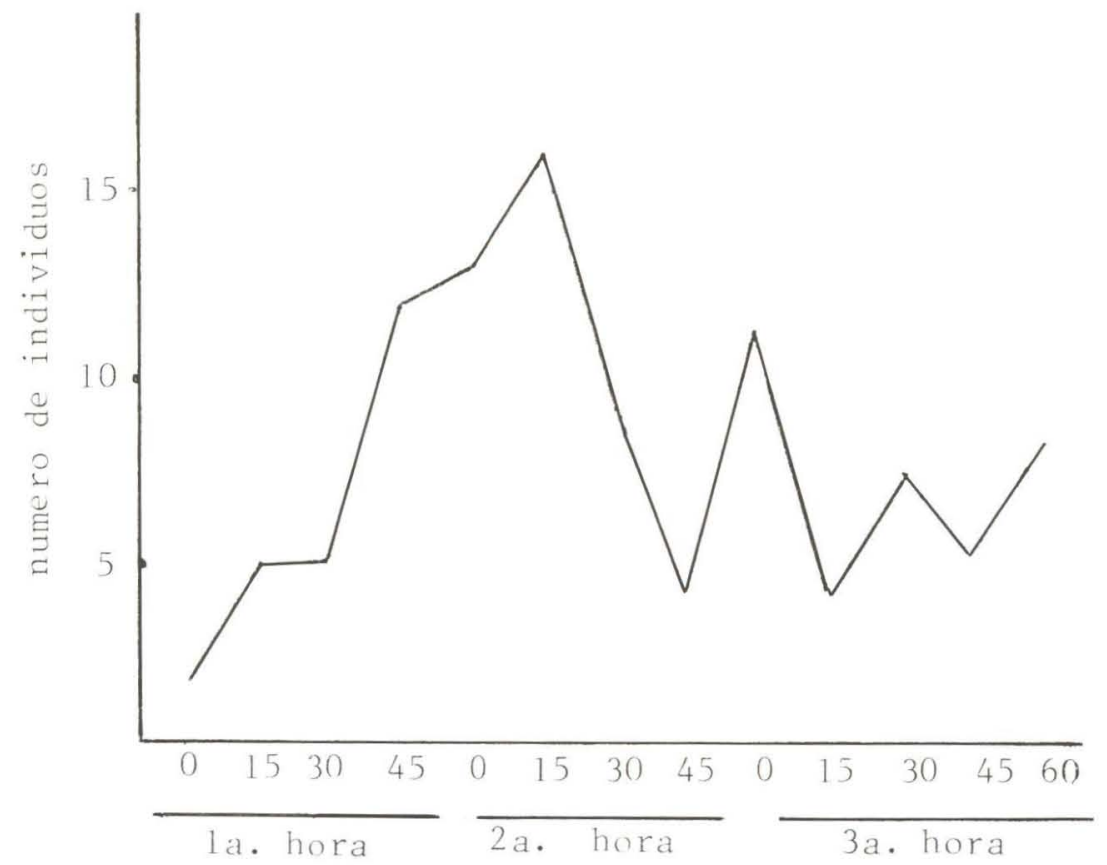

Fig. 3. Horário de captura dos quirópteros nas redes, durante as três primeiras horas após o escurecer.

\section{DISCUSSÃO}

Foram encontradas 15 espécies dentre 134 exemplares, perfazendo um percentual que justifica a afirmação de que uma floresta tropical, com seus inúmeros nichos, pode apresentar uma diversidade considerável (ODUM 1985). Dentre as espécies coletadas: uma era polinívora, sete exclusivamente insetívoras e sete frugívoras (Tab. I). Apesar do número de espécies frugívoras e insetívoras ser o mesmo, existe uma disparidade no número de indivíduos da amostra, sendo $75,3 \%$ de frugívoros e $20,4 \%$ de insetívoros. Tal fato decorre do método e do local das coletas. Através da utilização de redes armadas em uma altura de 0,5 a $2,0 \mathrm{~m}$ do solo como método de coleta, provocou-se a captura de um maior número de espécimes que voam ao nível das árvores frutíferas, diferentemente das insetívoras, que voam em diferentes estratos da floresta (HANDLEY 1967). O local onde foram realizadas as coletas, ou seja, em estradas que cortam a reserva passando por dentro da floresta, era quase sempre margeado por parte alterada com grande concentração de Mirtaceae, Solanaceae, Cecropiaceae e Piperaceae. Esta vegetação funciona como atrativo para os grupos frugívoros que assim contribuem na sucessão das comunidades vegetais, através da disseminação de suas sementes. Associações de morcegos com plantas pioneiras já foram mencionadas por diversos autores, entre eles HowELL \& BURCH (1974), FLEMING et al. (1972), 
Tabela III. Condições reprodutivas de fêmeas e machos coletados na Reserva Volta Redonda, Santa Catarina.

\begin{tabular}{|c|c|c|c|c|c|c|c|c|c|c|c|c|c|c|c|c|c|c|c|c|c|c|}
\hline \multirow{2}{*}{ Espécies } & \multicolumn{2}{|c|}{ Jan } & \multicolumn{2}{|c|}{ Fev } & \multicolumn{2}{|c|}{ Mar } & \multicolumn{2}{|c|}{ Abr } & Maı & \multicolumn{2}{|c|}{ Jun } & \multicolumn{2}{|c|}{ Jul } & \multicolumn{2}{|c|}{ Ago } & Set & \multicolumn{2}{|c|}{ Out } & \multicolumn{2}{|c|}{ Nov } & \multicolumn{2}{|l|}{ Dez } \\
\hline & $\mathrm{F}$ & M & $\mathrm{F}$ & M & $\mathrm{F}$ & M & F & M & M & $F$ & M & $\mathrm{F}$ & M & $\mathrm{F}$ & M & $F$ & $\mathrm{~F}$ & M & $\mathrm{F}$ & M & $\mathrm{F}$ & M \\
\hline Anoura caudifer & & & & & & $0 / 1$ & & & & & & $0 / 2$ & $0 / 1$ & & & & & & & & & \\
\hline Carollia perspicillata & & $0 / 1$ & & & & & & & & & $0 / 2$ & & & & & & & & & & & \\
\hline Sturnira lifium & & $1 \mathrm{~J}$ & $1 \mathrm{~J}$ & & & $0 / 2$ & & & & $0 / 1$ & & $\begin{array}{r}0 / 2 \\
1 \mathrm{~J}\end{array}$ & $0 / 1$ & & & $\begin{array}{ll}2 \mathrm{O} \\
2 \mathrm{~J}\end{array}$ & & $1 \mathrm{~J}$ & $1 \mathrm{~J}$ & & & \\
\hline Pygoderma bilabiatum & & & & & & & & & & $0 / 3$ & & $\begin{array}{r}0 / 2 \\
1 \mathrm{~J}\end{array}$ & $1 \mathrm{~J}$ & & & O I & 11 & & & & & \\
\hline Chiroderma doriae & & & & & $0 / 1$ & & $1 \mathrm{LO}$ & $0 / 10 /$ & $1 / 52 / 4$ & $40 / 1$ & & $0 / 1$ & & & & & & & & & & \\
\hline Artibeus fimbriatus & & & & & & $0 / 20$ & $2 / 10$ & $0 / 1$ & $0 / 1$ & & & & & & & & & & & & & \\
\hline Artibeus jamaicensis & & $1 / 1$ & & & & & & & & & & & $0 / 1$ & & & $1 \mathrm{~J} \mathrm{O}$ & 11 & & $1 / 1$ & & $2 / 2$ & \\
\hline Artibeus lituratus & $\begin{array}{c}1 / 1 \\
3 L\end{array}$ & $2 / 33$ & $3 / 3$ & & & & & $1 / 10 /$ & $132 / 0$ & $0 / 5$ & $0 / 1$ & $3 \mathrm{~J}$ & $2 \mathrm{~J} \mathrm{O}$ & $0 / 1$ & & $\begin{array}{r}1 / 03 / 5 \\
2\end{array}$ & $\begin{array}{l}5 \\
\mathrm{~J}\end{array}$ & & $1 / 1$ & & $\begin{array}{cc}0 / 1 & 2 \\
3 & 2\end{array}$ & $\begin{array}{r}2 / 5 \\
2 J\end{array}$ \\
\hline Myotis nigricans & $\begin{array}{c}1 / 2 \\
1 \mathrm{~J} \\
1 \mathrm{~L}\end{array}$ & $1 \mathrm{~J}$ & & $0 / 2$ & & $0 / 1$ & & & & $0 / 2$ & $0 / 4$ & & $0 / 1$ & & & $/ 21 /$ & $110 / 1$ & & & & & \\
\hline Myotis ruber & & & & & & & & & & & & & & & & $0 /$ & 12 & & & & & \\
\hline Eptesicus brasiliensis & & & & & & & & & & & $0 / 1$ & & & & & & & & & & & \\
\hline Lasiurus ega & & & & & & & & & & $0 / 2$ & & & & & & & & & & & & \\
\hline Lasiurus borealis & & & & & & & & & & & & & & $1 \mathrm{~J}$ & & & & & & & & \\
\hline Molossus molossus & & $1 / 1$ & & & & $0 / 1$ & & & & & & & & & & & & & & & & \\
\hline Molossus ater & & & & & & $1 / 1$ & & & & & & & & & & & & & & & & \\
\hline
\end{tabular}

(/) Representantes grávidas ou escrotados / indivíduos coletados; (J) jovem; (L) lactente.

CHARLES-DOMINIQUe (1986). Mas, de um modo geral, enquanto algumas espécies frugívoras voam baixo, na procura de diferentes alimentos, em horários distintos (MCNAB 1971; REIS 1984), evitando assim, competições os insetívoros caçam em diferentes alturas (HANDLEY 1967), como foi observado neste estudo.

Acredita-se que nenhum organismo está perfeitamente adaptado, porém, existem espécies que estão integradas em seus a mbientes em um maior número de dimensões (Pianka 1982), como mostram os resultados em relação aos frugívoros A. lituratus (35\% da amostra), S. lilium $(11,8 \%$ ) e o insetívoro $M$. nigricams $(14,8 \%)$ que tiveram ocorrência nas quatro estações do ano, mostrando um maior potencial adaptativo à variação de tempo e alimentação.

Observando a reprodução dos frugívoros, foi constatado um pico maior de fêmeas com prenhês positiva de novembro a fevereiro, de março a junho, observou-se um maior número de fêmeas não-grávidas. Todavia, como já foi estudado por Fleming et al. (1972); HeIthaus et al. (1975), há dois picos reprodutivos para frugívoros, mostrando um ciclo poliestro bimodal, com filhotes nascendo sempre fora da estação de inverno, fugindo de condições inóspitas com alimentação precária (REIS 1981). Quanto aos insetívoros, acredita-se que algumas espécies procriam em diferentes estações do ano, a fïm de diminuir a competição por alimentos, mantendo cada espécie o seu padrão específico (TADDEI 1976).

Em relação ao horário de atividade, os resultados mostram que pesquisas realizadas em três horas de coletas, após o escurecer, são suficientes para os estudos básicos de ecologia dos quirópteros, pela grande concentração de capturas durante esse tempo, confirmando assim dados de REIS (1981); REIS \& PERACCHI (1987). 
Já é evidente que muitos animais e vegetais encontram-se em vias de desaparecimento e que a responsabilidade dos estragos devastadores recai sobretudo na destruição dos habitats. Através da contribuição deste primeiro estudo sobre ecologia animal da Reserva Volta Velha, constatou-se, mais uma vez, a importância que os morcegos têm na formação e manutenção de matas, pela freqüência de algumas espécies frugívoras disseminadoras de sementes.

AGRADECIMENTOS. Agradecemos a confirmação das identificaçōes ao Prof. Dr. Adriano Lucio Peracchi da Universidade Federal Rural do Rio de Janeiro, ao Sr. Lucio Machado e ao CNPq por respaldar o presente estudo e a outros biólogos que ajudaram nas atividades de campo.

\section{REFERÊNCIAS BIBLIOGRÁFICAS}

BRoWN, J.H. 1968. Activity patterns of some neotropical bats.

J. Mammal. 49 (4): 754-757.

Carvalho, C.T. DE. 1960. Das visitas de morcegos às flores (Mammalia, Chiroptera). An. Acad. Bras. Ci. 32: 359-377.

Charles-DominiQue, P. 1986. Inter-relations between frugivorous vertebrates and pioneer plants: Cecropia, birds and bats in French Guyana, p. 119-135. In: A. Estrada \& T.H. Fleming (eds). Frugivorous and seed dispersal. Dordrecht, Junk Publishers, 392p.

Fabian, M.E.; S.M. Hartz \& T.H.A. Arigony. 1990. Alimentação de Tadarida brasiliensis (Geoffroy, 1824) na região urbana de Porto Alegre, RS, Brasil (Chiroptera, Molossidae). Rev. Bras. Biol. 50 (2): 387-392.

Fleming, T.H.; E.T. HoOper \& D.E. Wilson. 1972. Three Central American bat communites: Structure, reproductive cycle and movement patterns. Ecology 53: 555-569.

Glass, B.P. 1970. Feeding mechanisms of bats, p. 84-92. In: B.H. Slaughter \&W. WALTON (eds). About bats: a quiropteran symposium. Dallas, Southern Methodist Univ. Press, 399p.

Goodwin, G.G. \& A.M. Greenhall. 1961. A review of the hats of Trinidad and Tobago. Bull. Amer. Mus. Nat. Hist. 122 (3): 1-301.

Greenhall, G.G. 1966. The food of some Trinidad fruit bats. (Artibeus and Carollia). J. Agric. Soc., Trinidad and Tobago, 869: 1-23.

Greenhall, G.G. \& J.L. Paradiso. 1968. Bats and bat banding. Washington, U.S. Dept. Int. Fish and Wildlife Service, Bureau of Sport Fisheries and Wildlife 45p.

Griffin, D.R.; F.A. Webster \& C.R. Michael. 1960. The echolocation of flying insects by bats. Anim. Behav. 8: 141-154.

Handley JR., C.O. 1967. Bats of the canopy of an Amazonian forest. Atas do Simpósio sobre a biota Amazônica (Zoologia): 211-215.

Heithaus, E.R.; T.H. Fleming \& P.A Opler. 1975. Foraging paterns and resource intelization in seven fo hats in a seasond tropical forest. Ecology 56: 841-854. 
Howell, D.J. \& D. Burch. 1974. Food habits of some Costa Rican Bats. Rev. Biol. Trop. 21 (2): 281-294.

Humphrey, S.R. \& F.J. BonacCorso. 1979. Population and community ecology. Spec. Publ. Mus. Texas Tech. Univ. 16: 409-441.

Husson, A.M. 1962. The bats of Suriname. Zool. Verh., Leiden, 58: 1-282.

JONES, J.K. \& D.C. CARTER. 1977. Annotated checklist with keys to subfamilies and genera, p. 7-38. In: R.J. BAKER; J.K. Jones \& D. CARTER (eds). Biology of hats of the new world family Phillostomatidae. Part I. Spéc. Publ. Mus. Texas. Tech Univ., 10: 1-218.

KLEIN, R.H. 1978. Mapa fitogeográfico do Estado de Santa Catarina. Itajaí. Flora Ilustrada Catarinense, 24p.

KUNZ, T.H \& J.O. WHITAKER JR.. 1974. Feeding ecology of a temperate insetivorous bat (Myotis velifer). Ecology 55: 693-771.

LAVAL, R.K. 1970. Banding retturns and activity periods of some Costa Rican bats. Swter Nat 15 (1): 1-10.

MCNAB, B.K. 1971. The structure of tropical hat faunas. Ecology 52: 351-358.

Morrison, D.W. 1978. Lunar phobia in a neotropical fruit bat Artiheus jamaicensis. Anim. Behav. 26 (3): 852-853.

Odum, E.P. 1985. Fundamentos de ecologia. Lisboa, Fundação Calouste Gulbenkian, 3a. ed., 972p.

PianKa, E.R. 1982. Ecologia evolutiva. Barcelona, Omega, 927p.

PIJL, L. VAN DER. 1972. Principles of dispersal in higher plants. Berlim, Springer-Verlag, 2nd ed., $161 \mathrm{p}$.

PINE, R.H. 1968. Stomach contents of a free-tailed bat, Molossus ater. J. Mammal. 2 (2): 159-179.

REIS, N.R. DOS. 1981. Estudos ecológicos dos quirópteros de matas primárias e capoeiras da região de Manaus, Amazonas. Tese de Doutorado, não publicada, Instituto Nacional de Pesquisas da Amazônia, Fundação Universidade do Amazonas, Manaus, 242p.

- 1984. Estrutura de comunidade de morcegos na região de Manaus, Amazonas. Rev. Brasil. Biol. 44 (3): 247-254.

REIS, N.R. DOS \& A.L. PERACCHI. 1987. Quirópteros da região de Manaus, Amazonas, Brasil (Mammalia, Chiroptera). Bol. Mus. Par. Emílo Goeldi, Belém, 3 (2): 161-182.

Sazima, M. \& SazIMA, I. 1975. Quiropterofilia em Lafoensia pacario St. Hil (Lytraceae), na Serra do Cipó, Minas Gerais. Ci. Cult. 27 (4): 405-416. . 1978. Bat pollination of the Paswion flower, Passiflora mucronata in Southern Brazil. Biotropica 10 (2): 100-109.

Sazima, M.; M.E. Fabiam \& I. Sazima. 1982. Polinização de Luhéa speciosa (Tiliace'a) por Glossophaga soricina (Chiroptera, Phyllostomidae). Rev. Bras. Biol. 42 (3): 505-513.

SMrthe, N. 1970. Relationships between fruiting seasons and seed dispersal methods in a neotropical forest. Amer. Nat. 104 (935): 25-35.

TADDEI, V.A. 1976. The reproduction of some Phyllostomidae (Chiroptera) from 
the Northwestern region of the state of São Paulo. Bol. Zool. Univ. São Paulo 1: 313-30.

TAmsitT, J.R. \& D. VAldivieso. 1961. Notas sobre actividades noturnas y estados de reproducción de algunos quirópteros de Costa Rica. Rev. Biol. Trop. 9 (2): 219-225.

Uieda, W. \& J. Vasconcellos-Neto. 1985. Dispersão de Solamun sp. Solanaceae) por morcegos na região de Manaus, AM, Brasil. Revta bras. Zool. 2 (7): 449-458.

VieIRA, C.0. DA C. 1942. Ensaio monografico sobre os quirópteros do Brasil. Arq. Zool., São Paulo, 3 (8): 219-471.

VizotTo, L.T. \& TAdDEI, V.A. 1973. Chave para determinação de quirópteros brasileiros. Bol. Ci. 1: 1-72.

Vogel, S. 1969. Chiropterophile in derneotropuschen Flora. Neue Mittei lungen III. Flora Aht. B. 158: 289-323.

Recebido em 30.XI.1994; aceito em 20.IX.1995. 